\title{
Design of a Low Power 108-bit Conditional Sum Adder Using Energy Economized Pass-transistor Logic (EEPL)
}

Minkyu Song

Department of Semiconductor Science, Dongguk University 3-26, Phil-dong, Choong-ku, Seoul, 100-715, KOREA

TEL \& FAX: +82-2-260-3824

E-mail : mksong@cakra.dongguk.ac.kr

\begin{abstract}
In this paper, a novel 108-bit conditional sum adder(CSA) with Energy Economized Pass-transistor Logic(EEPL) is proposed. A new architecture is adopted, which is composed of seven modularized 16-bit CSA's and two separated CARRY Generation Block. In order to obtain a high speed operation, the CARRY Generation Blocks are separated from the modularized CSA. Further, a design technique based on EEPL is proposed to reduce the power consumption. With $0.65 \mathrm{\mu m}$ single poly, triple metal $3.3 \mathrm{~V}$ CMOS process, its operating speed is about $4.95 n s$ and the power consumption is reduced in comparison with that of the conventional adder.
\end{abstract}

\section{Keywords}

Conditional Sum Adder (CSA), Energy Economized Pass-transistor Logic(EEPL) Separated CARRY Generation Block 


\section{INTRODUCTION}

In recent years, higher-bit adders are key components which determine the system performance in the areas of digital signal processor(DSP), arithmetic logic unit(ALU), and so on. Therefore, the needs to design high speed and low power adders are becoming larger.

In this paper, an 108-bit conditional sum adder (CSA) with Energy Economized Pass-transistor Logic(EEPL) is proposed (Song, 1996). Pass-transistor Logic becomes popular in designing of VLSI systems. Due to the characteristics of the high speed operation and low power consumption, it is now widely used (Yano, 1996) (Sakurai, 1995). Based on EEPL, the proposed CSA has a high speed operation and a low power consumption. In order to obtain a high speed operation, a separated CARRY Generation Block is proposed and designed in this study. In comparison with the conventional adder (Ohkubo, 1995), it has a faster operating speed and lower power consumption. With $0.65 \mu \mathrm{m}$ single poly, triple metal 3.3V CMOS process, its operating speed is about $4.95 n s$ and the power consumption is reduced in comparison with that of the conventional adder.

In Section II, a proposed architecture of the 108-bit CSA is discussed. We explain how it is designed and the advantage of the architecture. Further, the circuit design technique based on EEPL will be also discussed. In Section III, the separated CARRY Generation Block is described. The characteristics of the separated CARRY Generation Block is mainly discussed. In section IV, simulation results and other characteristics are described. Finally, we summarize the results in section $\mathrm{V}$.

\section{II . ARCHITECTURE OF THE PROPOSED ADDER}

The proposed architecture of a Conditional Sum Adder(CSA) with a separated CARRY Generation Block is shown in Figure 1. It is composed of seven modularized 16-bit CSA's and two separated CARRY Generation Block. In order to obtain a high speed operation, the CARRY Generation Blocks are separated from the modularized CSA. Before the carry propagation of each modularized CSA is transferred to the final stage, the block carry propagation(BCi , BCiB) of the separated CARRY Generation Block drives the final stage. It will be discussed later.

Each modularized 16-bit CSA is composed of both Pre C\&S Generation Block and SUM Generation Block. The role of Pre C\&S Generation Block is generating both preliminary CARRY and SUM signals. In order to obtain the final results, it is advantageous to handle the preliminary signals. It will be discussed in this section. At the SUM Generation Block the desired results are obtained. Using some kinds of multiplexers, the output can be obtained in a short time. Based on this architecture, the total delay is reduced by about two MUX equivalent delay time in comparison with that of the conventional one (Ohkubo, 1995). 


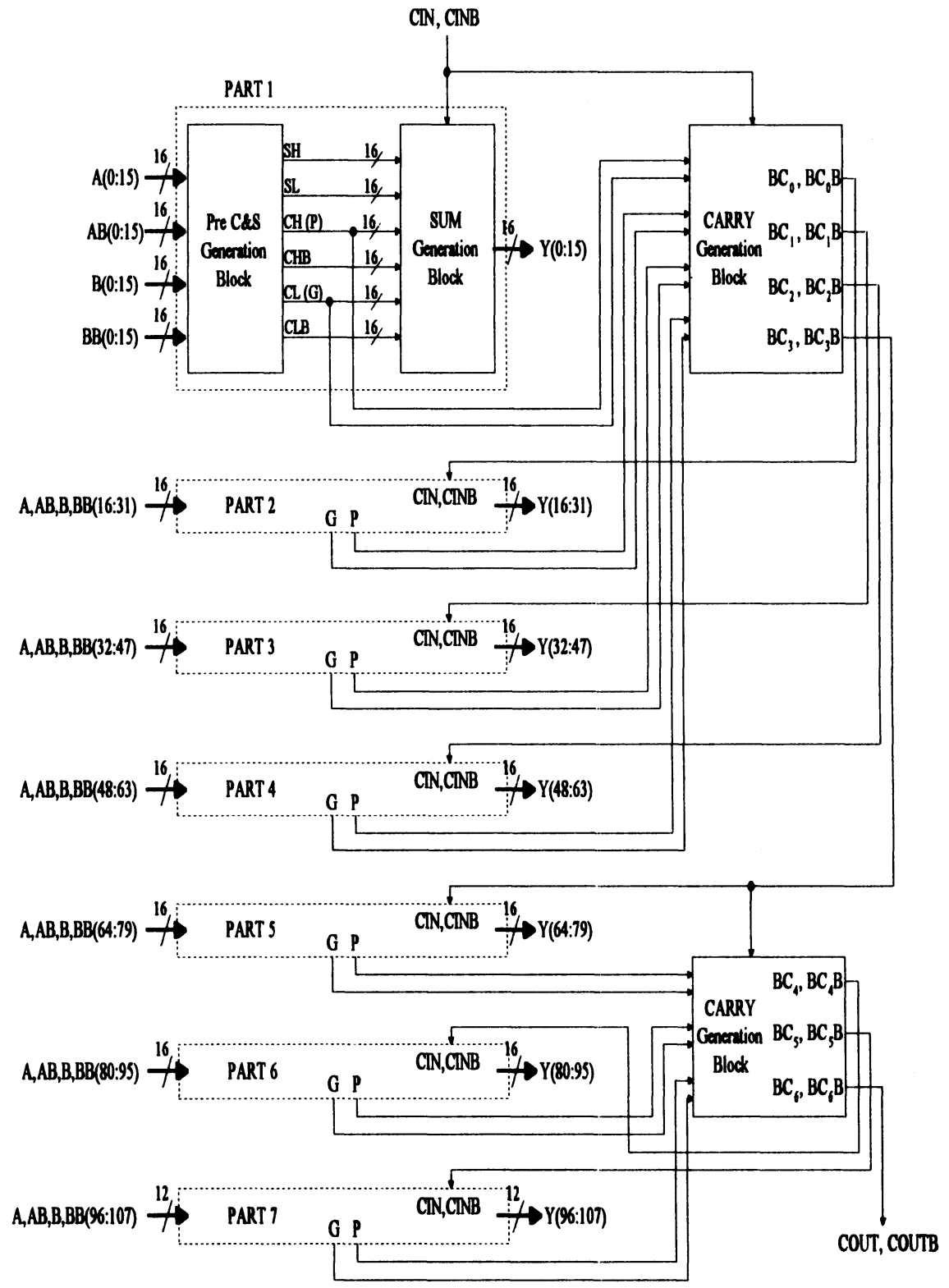

Figure 1 The Architecture of the 108-bit Conditional Sum Adder(CSA). It is composed of seven 16-bit modularized CSA's (16-b x 7 CSA) and two high speed Carry Generation Blocks. Before the carry propagation of each modularized CSA transfers to the final stage, the carry propagation of the high speed Carry Generation Block drives the end of stage. Therefore, the total delay is shorter by about two MUX equivalent delay than that of the conventional one. 


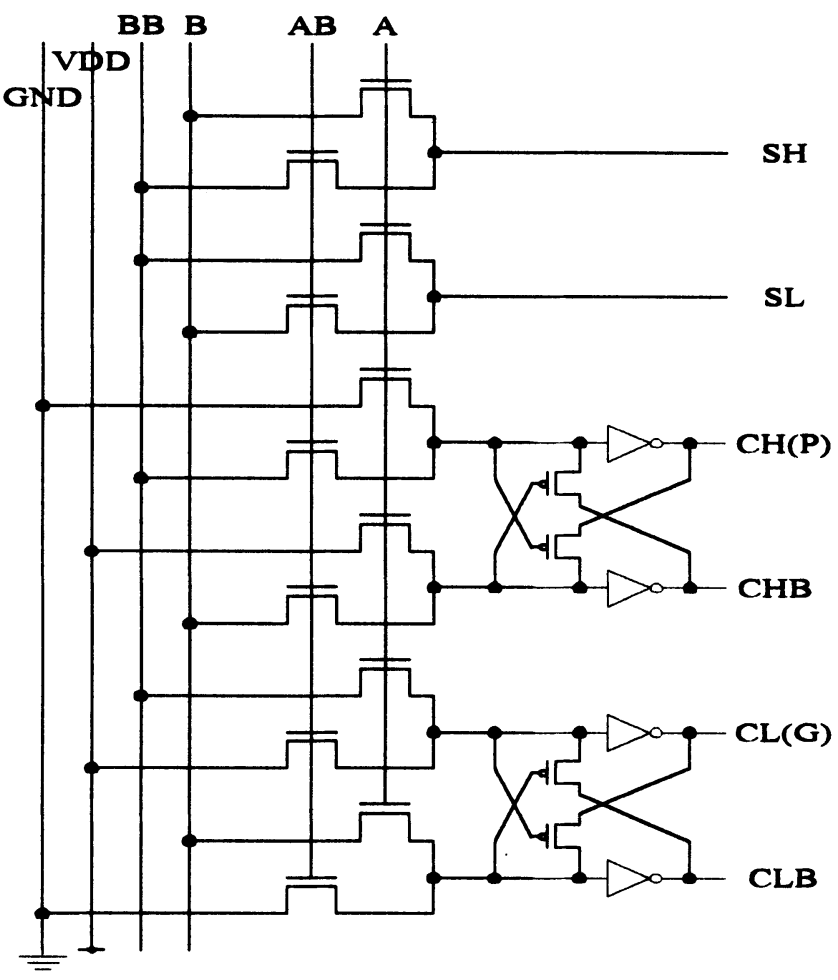

Figure 2 Circuit Diagram of the Pre C\&S Generation Block. $\mathrm{CH}$ is equivalent to the Propagate Signal of the two input and CL is equivalent to the Generate Signal of the two input:

Figure 2 shows the circuit diagram of the Pre C\&S Generation Block shown in Figure 1. In this block, the equations of each signal are as followings;

$$
\begin{aligned}
& S H=A \cdot B+\bar{A} \cdot \bar{B} \quad(X N O R) \\
& S L=\bar{A} \cdot B+A \cdot \bar{B} \quad(X O R) \\
& C L=G+P \cdot C_{-1}=G=A \cdot B \\
& C H=G+P \cdot C_{-1}=G+P=P=A+B
\end{aligned}
$$

Except for the signals $S H$ and $S L$, other signals are generated by EEPL. $S H$ and $S L$ are sent to the input of the SUM Generation Block, and the level of the signals are restored in this block. This is because it is efficient to connect level restoration circuit at the optimal positions (Song, 1996). Further, the metal routing is going to be complicated, if there exist so many level restoration circuits. 
Figure 3 shows the block diagram of the SUM Generation Block. In the figure, the desired output is obtained from a principle of a simple CSA and proposed equations as followings

$$
\begin{aligned}
S_{j} & =X_{j} \bullet\left(\overline{G_{j-1}+P_{j-1} C_{j-2}}\right)+\overline{X_{j}} \bullet\left(G_{j-1}+P_{j-1} C_{j-2}\right) \\
& =\bar{X}_{j} \quad\left(\text { when } G_{j-1}+P_{j-1}=P_{j-1}=1\right) \\
& =X_{j} \quad\left(\text { when } G_{j-1}+P_{j-1}=P_{j-1}=0\right) \\
S L_{j} & =X_{j} \bullet\left(\overline{G_{j-1}+P_{j-1} C_{j-2}}\right)+\overline{X_{j}} \bullet\left(G_{j-1}+P_{j-1} C_{j-2}\right) \\
& =\bar{X}_{j} \quad\left(\text { when } G_{j-1}=1\right) \\
& =X_{j} \quad\left(\text { when } G_{j-1}=0\right)
\end{aligned}
$$

Due to the characteristics of the conditional sum adder, the previous data located two stages before are able to be used now. Thus the operating speed is faster than that of the conventional one. This is one of the main idea in this paper. Figure 4 shows the circuit diagrams of each multiplexer used in Figure 3. MS means a multiplexer with single input, MSL means multiplexer with single input and level restoration circuit, MD means multiplexer with double inputs, and MDL means multiplexer with double inputs and level restoration circuit. When the output of a multiplexer drives the next gate of a multiplexer, we have to use multiplexers with a level restoration circuit. This is because it is necessary to drive the gate input within a full range. However, when the output of a multiplexer just drives the input of a multiplexer, we do not need to connect a level restoration circuits. In the same way, the best optimized multiplexer is chosen and designed according to the role and location of each multiplexer as shown in Fig.3.

Fig.5 shows the SPICE simulation results of each multiplexer. The delay time of MDL is faster than that of MSL. This is because the delay time of a level restoration circuit based on EEPL is faster than that of the circuit based on the simple inverter shown in Figure 4(b). Assuming the signals of CIN and CINB from Figure 1 are driven faster by the block of the separated CARRY Generation Block in comparison with the carry propagation of the modularized CSA, the propagation delay of 108-bit adder is equivalent to that of ten MDL in cascade. This is because there exists one equivalent MDL in Pre-C\&S Generation Block, eight equivalent MDL in 16-bit CSA, and one MSL driven by CIN in the final stage. It is simply calculated in Figure 3. The critical path is composed of both eight vertical MDL and one MSL as shown in Figure 3. In the conventional CSA, the propagation delay time of the 108-bit adder is equivalent to that of twelve MDL in cascade (Ohkubo, 1995). Therefore, the propagation delay time of the proposed adder is reduced by about two equivalent MDL delay in comparison with that of the conventional adder. In section III, we describe the design of the separated CARRY Generation Block. 


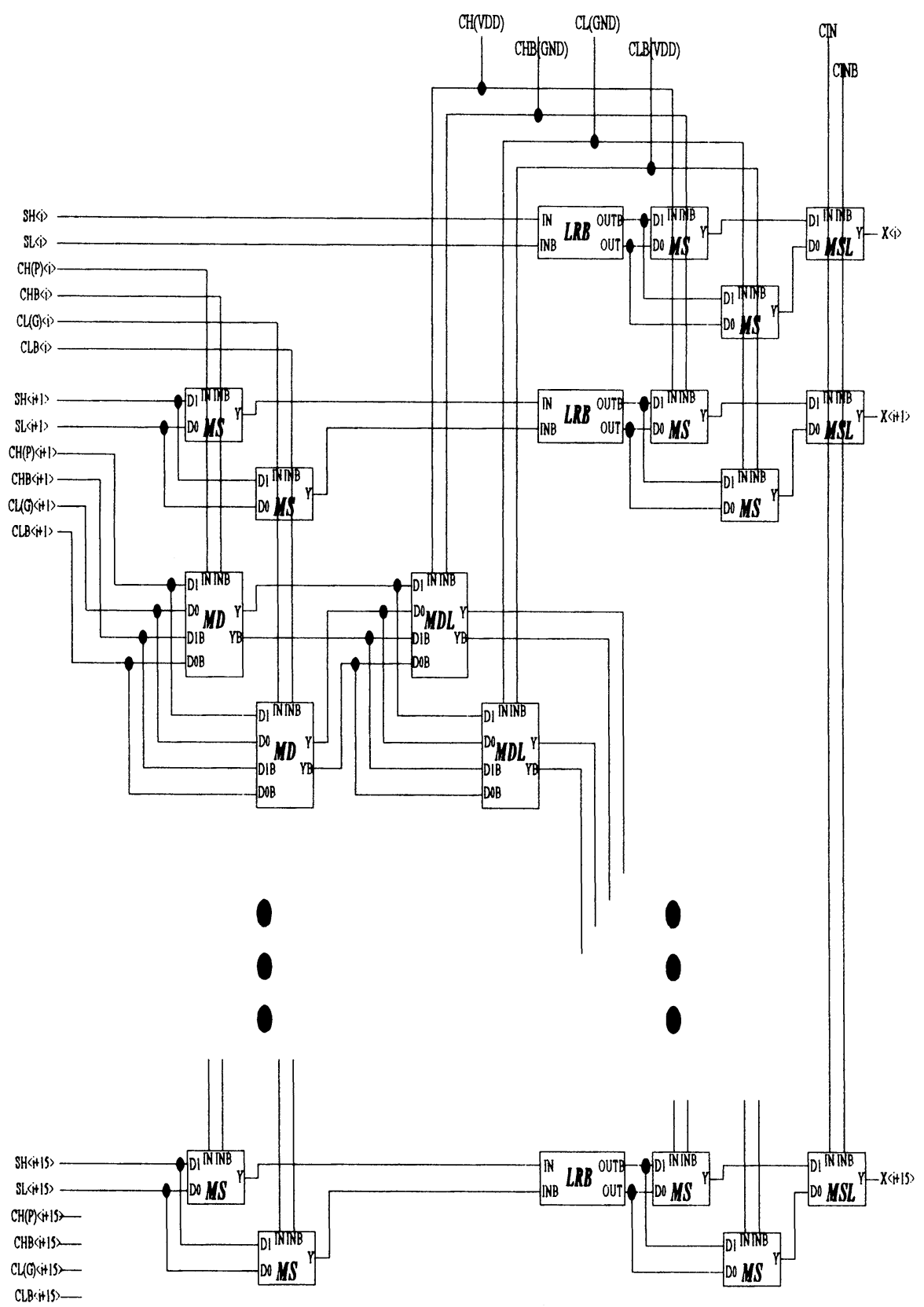

Figure 3 Block Diagram of the SUM Generation Block 


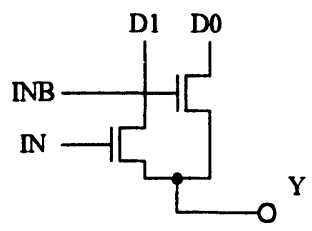

(a)MS

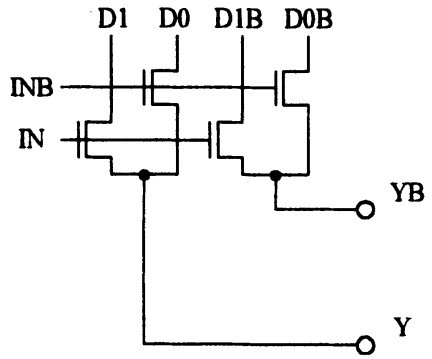

(c)MD

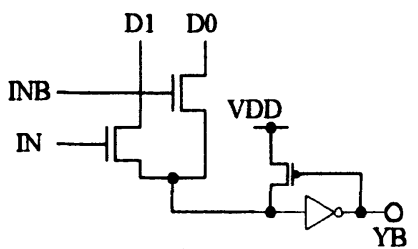

(b)MSL

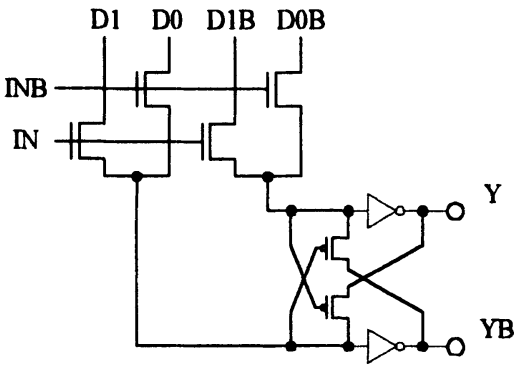

(d)MDL

Figure 4 Circuit Diagram of Each Multiplexer. According to the location and role of each MUX, they were optimized. (a) MS(Multiplexer with Single input) (b)MSL(Multiplexer with Single input and Level restoration block) (c)MD (Multiplexer with Double input) (d)MDL (Multiplexer with Double input and Level restoration block)

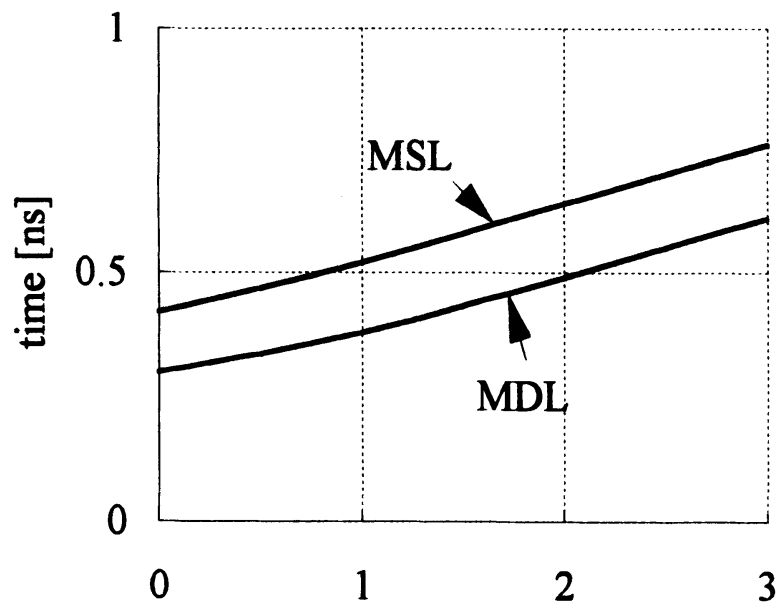

Fan Out

Figure 5 Comparison of Each Multiplexer. The delay time of MDL is faster than that of MSL 


\section{DESIGN OF THE SEPARATED CARRY GENERATION BLOCK}

Block diagram of the separated CARRY Generation Block is shown in Figure 6. It is designed in order to make the operating speed higher. It is composed of both CLA1 and CLA2 whose circuit diagrams are shown in Figure 7 and Figure 8, respectively. CLA1 is designed on the basis of the theory of group sum and group carry. Each CLAl is composed of 4-bit group. If we design CLAl with the conventional CMOS circuits shown in Figure 7, we can reduce the propagation delay by about two MUX equivalent time in comparison with that of the conventional one. After two CLA1 is connected in cascade, the output of it is transferred to CLA2. CLA2 is composed of both CLA1 and MDL as shown in Figure 8. Therefore, the total propagation delay time of the separated CARRY Generation Block is equivalent to three CLAl and two MDL. In other words, that is equivalent to eight MUX delay in series. In comparison with that of the SUM Generation Block, that of the separated CARRY Generation Block is faster by about two equivalent MUX delay.

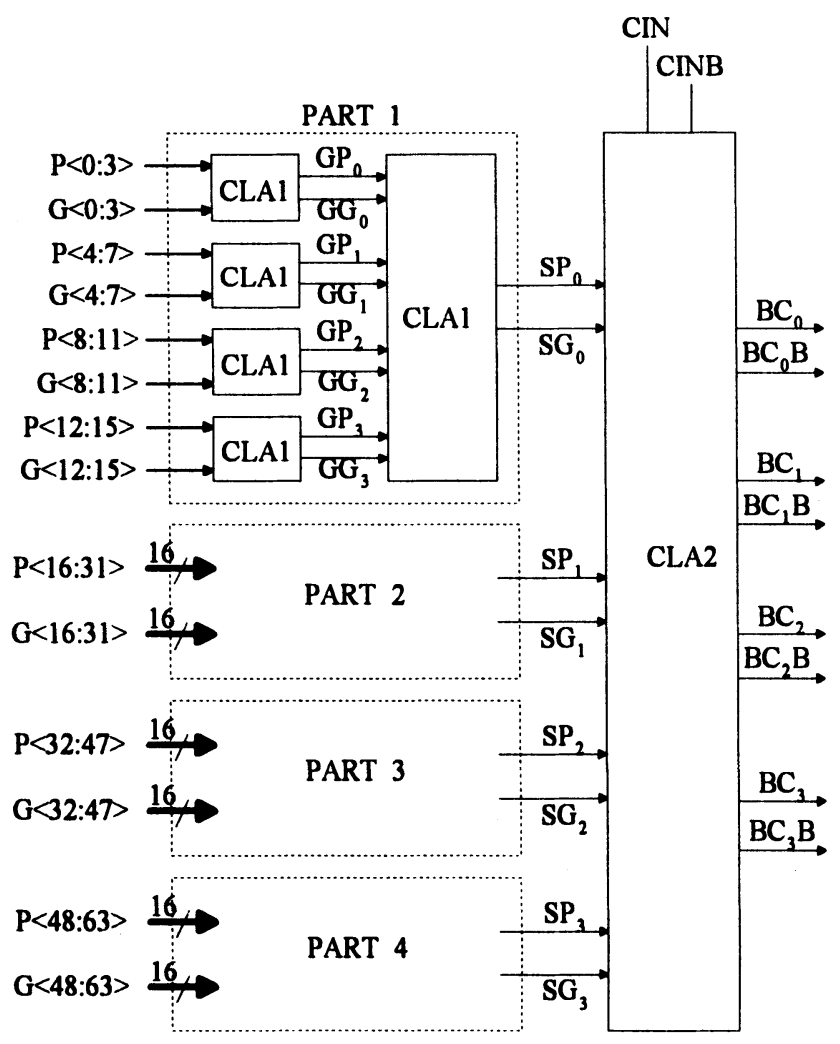

Figure 6 Block Diagram of the Proposed High Speed CARRY Generation Block. 


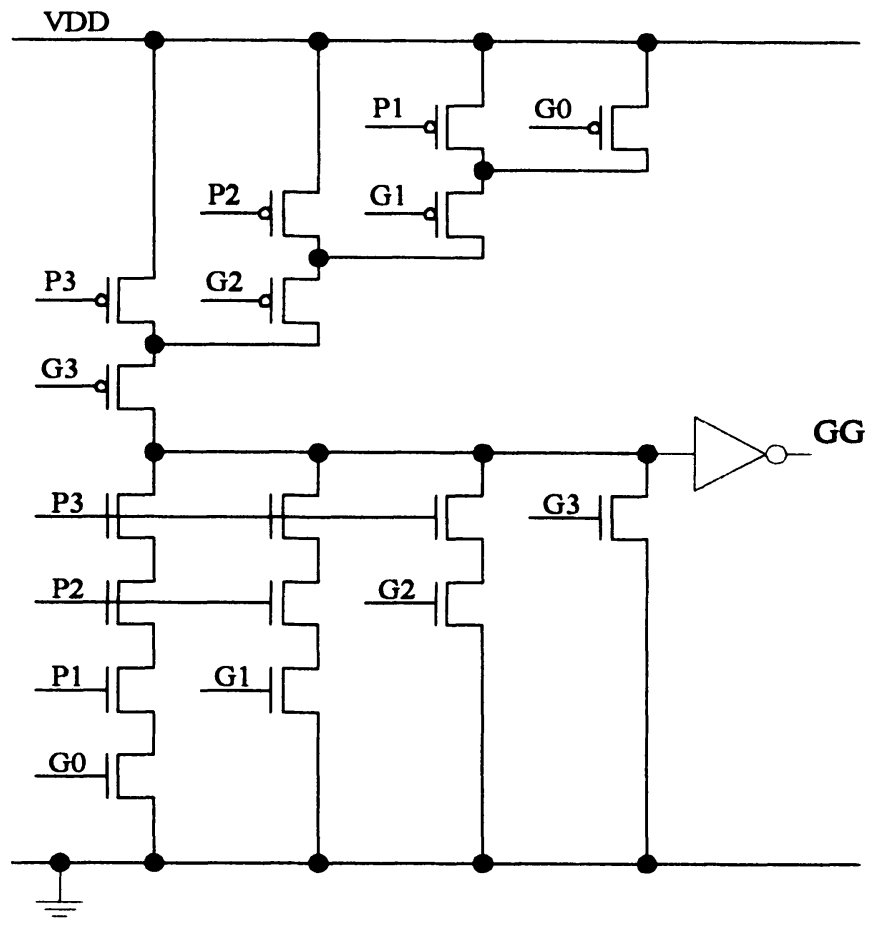

(a)

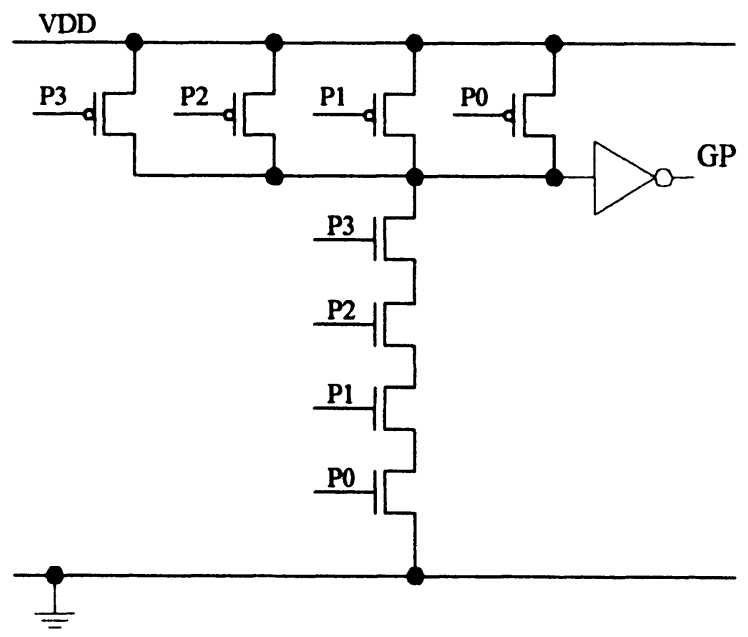

(b)

$\begin{array}{llll}\text { Figure } 7 \text { Circuit Diagram of CLAl. (a)Group Generate(GG) (b)Group } & \end{array}$ Propagate(GP) 


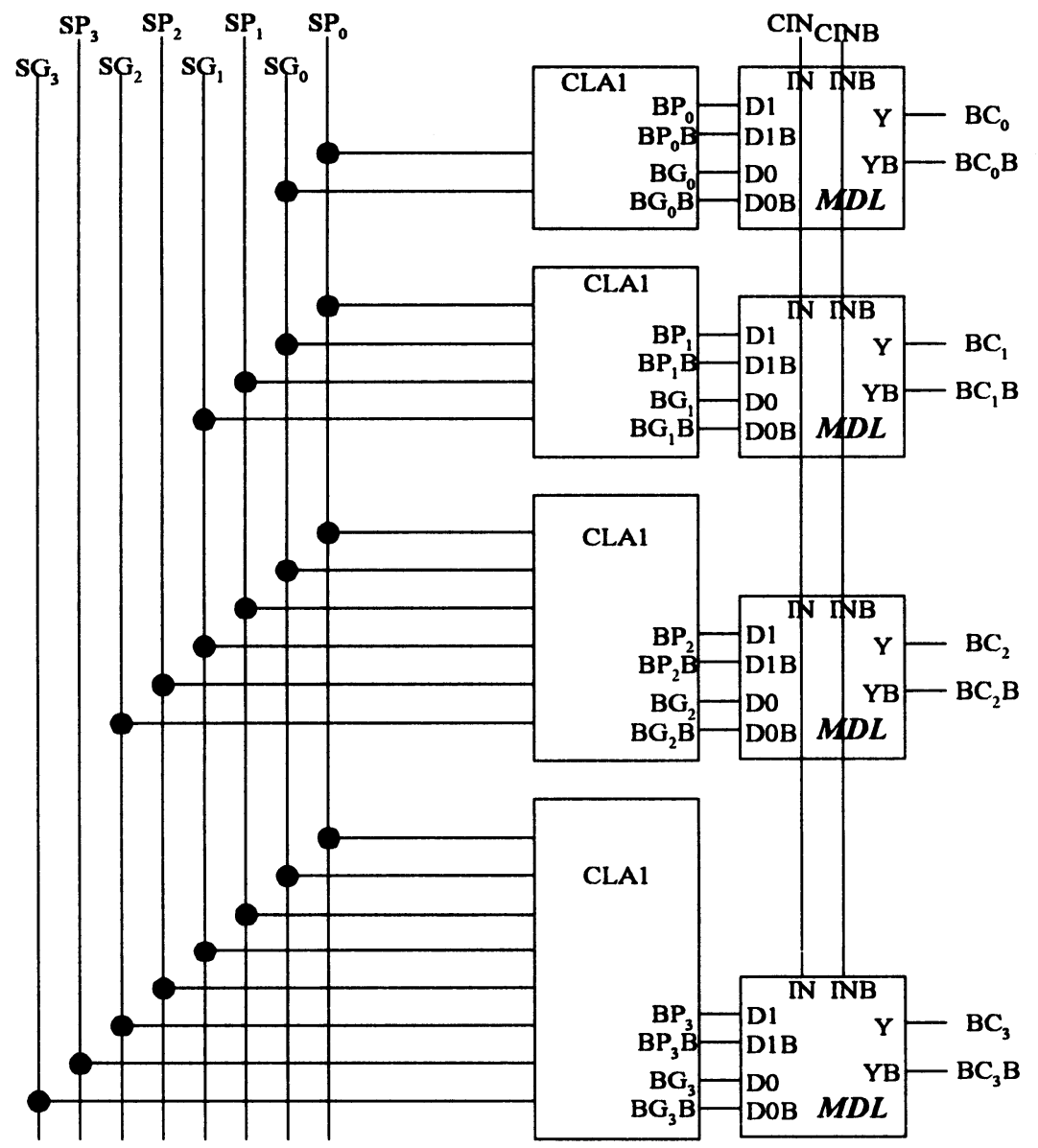

Figure 8 Block Diagram of the CLA2. It is composed of both CLA1 and MDL.

\section{RESULTS}

Figure 9 shows the SPICE simulation results of the proposed 108-bit adder. The delay of the carry propagation is faster than that of the sum propagation. The delay is about $4.95 \mathrm{~ns}$. In the figure, $\mathrm{BC} 7$ means the final carry and $\mathrm{X}<107>$ means the final sum result of the 108-bit adder. As the signal of the carry propagation is coming faster, the critical path of the 108-bit CSA is shorter than that of the conventional adder. Further, the power consumption of the proposed adder is smaller than that of the conventional one. The SPICE model parameters and experimental results have been carried out with $0.65 \mathrm{\mu m}$ triple metal single poly 3.3V CMOS technology. 


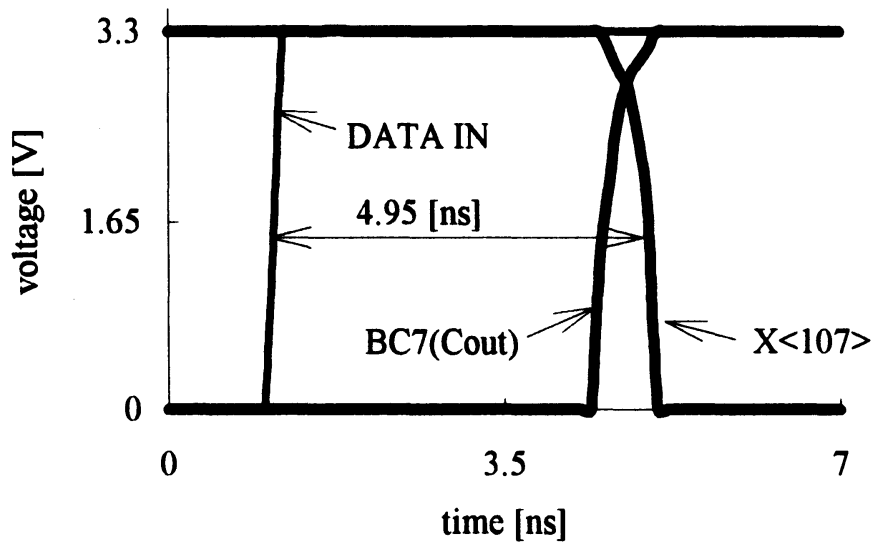

Figure 9 SPICE Simulation Results of the Proposed 108-bit Adder. The delay of the carry propagation is faster than that of the sum propagation.

\section{CONCLUSIONS}

A low power 108-bit Conditional Sum Adder with Energy Economized Passtransistor Logic (EEPL) was proposed. It consumed less power in comparison with that of the conventional one. This was because the proposed adder was designed on the basis of EEPL. In order to obtain a high speed operation, an architecture with a separated high speed CARRY Generation Block was adopted. With $0.65 \mu \mathrm{m}$ single-poly triple-metal CMOS process at $3.3 \mathrm{~V}$ power supply, the propagation delay of the proposed adder was about $4.95 \mathrm{~ns}$. It will be an useful technique to design a high speed and low power digital signal processor and other applications.

\section{REFERENCES}

M.Song, et.al., "Design Methodology for High Speed and Low Power Digital Circuits with Energy Economized Pass-transistor Logic(EEPL)", in Proc. IEEE ESSCIRC'96, pp.120-123, Sep., 1996.

K.Yano, et.al., "Top-Down Pass-Transistor Logic Design", IEEE J. of Solid-State Circuits, vol.31, no.6, pp.792-803,Jun.,1996.

T.Sakurai, et.al.,"Low-Power Circuit Design for Multimedia VLSI", in Proc. IEEE ICVC'95, pp.37-42, Oct., 1995.

N.Ohkubo, et.al., "A 4.4ns CMOS 54x54-b Multiplier Using Pass-Transistor Multiplexer", IEEE J. of Solid-State Circuits, vol.30, no.3, pp.251-257, Mar.,1995. 


\section{BIOGRAPHY}

\section{Minkyu Song}

He was born in Seoul, Korea, in 1963. He received the B.S., M.S., and Ph.D. degrees in Electronics Engineering from Seoul National University, Korea in 1986, 1988, and 1993, respectively. From 1993 to 1994, he was a researcher at the University of Tokyo, Japan where he worked in the area of low power VLSI design including CMOS analog circuits and digital circuits. From 1995 to 1996, he was a senior researcher at the team of ASIC core design, Samsung Electronics Co. Ltd., Kiheung, Korea where he worked in the area of low power CMOS analog and digital circuits. He is currently working as an associate professor at the department of Semiconductor Science, Dongguk University, Korea. 